\title{
TRANSFORMATION GROUPS ON COHOMOLOGY PROJECTIVE SPACES(1)
}

\author{
BY
}

J. C. SU

1. Introduction. Let $G$ be a topological transformation group on a compact Hausdorff space $Y$ and $F(G ; Y)$ its fixed point set. The present paper is devoted to the study of the cohomology structure of $F(G ; Y)$ in the following three cases:

(1) $G$ is the group $Z_{2}$ of integers modulo 2 and $Y$ has the $\bmod 2$ cohomology ring of the real projective $n$-space.

(2) $G$ is the group $Z_{p}$ of integers modulo $p$, where $p$ is an odd prime, and $Y$ has the $\bmod p$ cohomology structure of the lens $(2 n+1)$-space $\bmod p$.

(3) $G$ is the circle group $S^{1}$ (the group of reals mod 1) and $Y$ has the integral cohomology ring of the complex projective $n$-space.

For the sake of simplicity, we shall call $Y$ a cohomology real projective $n$ space or a cohomology lens $(2 n+1)$-space $\bmod p$ or a cohomology complex projective $n$-space if its cohomology structure is that described in (1) or (2) or (3). (Formal definition of these notions will be given later.)

The study of the problem proposed above is motivated by two recent theorems obtained separately by P. A. Smith and C. T. Yang. In [6], Smith proved that if $Z_{2}$ acts effectively on the real projective $n$-space, then the fixed point set is either empty, or it has exactly two components $C_{1}$ and $C_{2}$, where each $C_{i}$ is a cohomology real projective $n_{i}$-space, $i=1,2$, and $n_{1}+n_{2}=n-1$. Later in an unpublished work, Yang proved that if $S^{1}$ acts differentiably on the complex projective $n$-space, then the fixed point set is nonempty, has at most $n+1$ components, say $C_{1}, \cdots, C_{k}, k \leqq n+1$, where each $C_{i}$ is a cohomology complex projective $n_{i}$-space, $i=1,2, \cdots, k$, and $n_{1}+n_{2}+\cdots+n_{k}=n-k+1$. Our main purpose is to show that, under the more general setting of (1) and (3), essentially the same conclusions obtained by Smith and Yang still hold true. We also include a study of case (2), which is the natural counter part of case (1) when $p$ is odd.

All topological spaces considered in this paper are assumed to be compact Hausdorff. For such a space $X, H^{*}(X ; L)=\sum_{n=0}^{\infty} H^{n}(X ; L)$ will denote the Alexander-Spanier-Wallace cohomology ring with coefficient domain $L$. Let $G$

Presented to the Society October 28, 1961 under the title Transformation groups on cohomology lens spaces and cohomology complex projective spaces; received by the editors February 23, 1962.

(1) This research was sponsored by the U. S. Army Research office under contract No. DA 36-034-ORD-2970, and is part of a doctoral dissertation written at the University of Pennsylvania under Professor C. T. Yang. 
be a transformation group on a space $X$. For each $x \in X$ the closed subgroup $G_{x}=\{g \in G \mid g x=x\}$ of $G$ is called the isotropic subgroup at $x$ and the subset $G(x)=\{g x \in X \mid g \in G\}$ of $X$ is called an orbit. The action is said to be free (or $G$ acts freely on $X$ ) if $G_{x}=e$ (the identity subgroup of $G$ ) for all $x \in X$. The set $X / G=\{G(x) \mid x \in X\}$ endowed with the usual quotient topology is called the orbit space. The map (the word map will always mean a continuous map in this paper) sending $x \in X$ to $G(x) \in X / G$ is called the canonical projection and will be denoted by $\pi: X \rightarrow X / G$. We call $X$ a cohomology $n$-sphere over $L$ if $H^{*}(X ; L)$ $=H^{*}\left(S^{n} ; L\right)$, where $S^{n}$ denotes the $n$-sphere. If $L=Z_{p}$, we also call $X$ a cohomology $n$-sphere $\bmod p$. If $L=Z$ (the group of integers), we also call $X$ an integral cohomology $n$-sphere. As usual, empty space is regarded as cohomology (-1)-sphere over $L$.

2. Cohomology real projective spaces and cohomology lens spaces. Throughout this section, the coefficient domain $L$ for cohomology will be the field $Z_{p}$ of characteristic $p \neq 0$. We adopt the convention that $H^{*}(X)$ shall mean $H^{*}\left(X ; Z_{p}\right)$ for $p \neq 2$ and for $p=2$ the coefficient domain $Z_{2}$ shall be indicated out explicitly. A space $Y$ is called a cohomology real projective $n$-space if the ring $H^{*}\left(Y ; Z_{2}\right)$ is given by

$$
H^{*}\left(Y ; Z_{2}\right)=Z_{2}[x] /\left(x^{n+1}\right), \text { degree } x=1,
$$

where $Z_{2}[x]$ is the polynomial ring with coefficients in $Z_{2}$ and $\left(x^{n+1}\right)$ is the ideal generated by $x^{n+1}$. $Y$ is called a cohomology lens $(2 n+1)$-space $\bmod p, p \neq 2$, if the ring $H^{*}(Y)$ is given by

$$
H^{*}(Y)=\Lambda[a] \otimes Z_{p}[x] /\left(x^{n+1}\right), \quad \text { degree } a=1, \text { degree } x=2,
$$

where $\Lambda[a]$ is the exterior algebra generated by $a$ over $Z_{p}, Z_{p}[x]$ is the polynomial ring with coefficients in $Z_{p}$ and $\left(x^{n+1}\right)$ is the ideal generated by $x^{n+1}$. In addition, we also require that $\beta(a)=x$, where $\beta: H^{1}(Y) \rightarrow H^{2}(Y)$ is the Bockstein homomorphism. The following two propositions are well known.

Proposition 2.3. If $Z_{2}$ acts freely on a cohomology $n$-sphere $\bmod 2 X$, then $X / Z_{2}$ is a cohomology real projective $n$-space.

Proposition 2.4. If $Z_{p}(p \neq 2)$ acts freely on a cohomology $(2 n+1)$-sphere $\bmod p X$, then $X / Z_{p}$ is a cohomology lens $(2 n+1)$-space $\bmod p$.

Much more interesting is their converse. We have

THEOREM 2.5. If $Z_{2}$ acts freely on a connected space $X$ such that $X / Z_{2}$ is a cohomology real projective $n$-space, then $X$ is a cohomology $n$-sphere mod 2.

Proof. Using Smith's special cohomology [1, p. 41], we have the Smith exact sequence which in the case of free action of $Z_{2}$ takes the following form. 


$$
\begin{aligned}
0 \rightarrow H^{0}\left(X / Z_{2} ; Z_{2}\right) & \stackrel{\pi^{*}}{\rightarrow} H^{0}\left(X ; Z_{2}\right) \rightarrow H^{0}\left(X / Z_{2} ; Z_{2}\right) \stackrel{\delta}{\rightarrow} H^{1}\left(X / Z_{2} ; Z_{2}\right) \rightarrow \cdots \\
& \rightarrow H^{k-1}\left(X / Z_{2} ; Z_{2}\right) \stackrel{\delta}{\rightarrow} H^{k}\left(X / Z_{2} ; Z_{2}\right) \stackrel{\pi^{*}}{\rightarrow} H^{k}\left(X ; Z_{2}\right) \\
& \rightarrow H^{k}\left(X / Z_{2} ; Z_{2}\right) \rightarrow \cdots
\end{aligned}
$$

where $\pi^{*}: H^{k}\left(X / Z_{2} ; Z_{2}\right) \rightarrow H^{k}\left(X ; Z_{2}\right)$ is the homomorphism induced by the canonical projection $\pi: X \rightarrow X / Z_{2}$. Since $X$ is connected, $H^{0}\left(X ; Z_{2}\right)=Z_{2}$ and hence $\pi^{*}: H^{0}\left(X / Z_{2} ; Z_{2}\right) \rightarrow H^{0}\left(X ; Z_{2}\right)$ must be an isomorphism. This implies that $\pi^{*}: H^{1}\left(X / Z_{2} ; Z_{2}\right) \rightarrow H^{1}\left(X ; Z_{2}\right)$ is trivial. The ring structure imposed on $H^{*}\left(X / Z_{2} ; Z_{2}\right)$ then implies that $\pi^{*}: H^{k}\left(X / Z_{2} ; Z_{2}\right) \rightarrow H^{k}\left(X ; Z_{2}\right)$ is trivial for all $k \geqq 1$. The theorem follows immediately from the exactness of (1).

THEOREM 2.6. If $Z_{p}(p \neq 2)$ acts freely on a connected space $X$ such that $X / Z_{p}$ is a cohomology lens $(2 n+1)$-space mod $p$, then $X$ is a cohomology $(2 n+1)$ sphere $\bmod p$.

We need some preliminary considerations. The action of $Z_{p}$ on $X$ induces an action of $Z_{p}$ on $H^{*}(X)$. More precisely, let $T$ be a generator of $Z_{p}$; then the induced homomorphism $T^{*}: H^{*}(X) \rightarrow H^{*}(X)$ satisfies $T^{* p}=1$ (the identity homomorphism) and hence defines an action of $Z_{p}$ on $H^{*}(X)$. We can therefore talk about the functor $H^{*}\left(Z_{p} ; H^{*}(X)\right)$, the cohomology of the group $Z_{p}$ with coefficients in $H^{*}(X)$. Let $\tau^{*}=1-T^{*}, \sigma^{*}=\sum_{i=0}^{p-1} T^{* i}$ and $H^{*}(X)^{0}=\operatorname{ker} \tau^{*}$, we have [2]

$$
H^{s}\left(Z_{p} ; H^{*}(X)\right)= \begin{cases}H^{*}(X)^{0} & \text { if } s=0, \\ \operatorname{ker} \tau^{*} / \operatorname{Im} \sigma^{*} & \text { if } s=2 k, k>0, \\ \operatorname{ker} \sigma^{*} / \operatorname{Im} \tau^{*} & \text { if } s=2 k+1, k \geqq 0 .\end{cases}
$$

Moreover, $H^{*}\left(Z_{p} ; Z_{p}\right)=\sum_{s=0}^{\infty} H^{s}\left(Z_{p} ; Z_{p}\right)$ has a ring structure which can be described as

$$
H^{*}\left(Z_{p} ; Z_{p}\right)=\Lambda[a] \otimes Z_{p}[x] \text {, degree } a=1 \text {, degree } x=2,
$$

where $\Lambda[a]$ and $Z_{p}[x]$ are the same as in (2.2). Furthermore, we also have $\beta(a)=x$, where $\beta: H^{1}\left(Z_{p} ; Z_{p}\right) \rightarrow H^{2}\left(Z_{p} ; Z_{p}\right)$ is the Bockstein homomorphism. The following two lemmas can be easily established.

LEMMA 2.9. If $\sigma^{*}=0$ and $H^{k}(X)^{0}=0$, then $H^{k}(X)=0$.

LEMMA 2.10. If $\sigma^{*}=0$ and $\operatorname{dim} H^{k}(X)^{0}=1$, then $\operatorname{dim} H^{k}(X) \leqq p-1$.

Proof of (2.6). We shall only give a proof for the case $n>0$. Since $Z_{p}$ acts freely on $X$, there is the Leray-Cartan spectral sequence [1] $\left(E_{r}\right)$ whose $E_{2}$-term is given by $E_{2}^{s, t}=H^{s}\left(Z_{p} ; H^{t}(X)\right)$ and whose $E_{\infty}$-term is associated with $H^{*}\left(X / Z_{p}\right)$. As $E_{2}^{s, t}=0$ when either $s$ or $t$ is negative, we have the exact sequence for low dimensions [2]

$$
0 \rightarrow E_{2}^{1,0} \stackrel{\phi_{1}}{\longrightarrow} H^{1}\left(X / Z_{p}\right) \stackrel{\pi^{*}}{\longrightarrow} E_{2}^{0,1} \stackrel{d_{2}}{\longrightarrow} E_{2}^{2,0} \stackrel{\phi_{2}}{\longrightarrow} H^{2}\left(X / Z_{p}\right) .
$$


Since $X$ is connected, $E_{2}^{*, 0}=\sum_{s=0}^{\infty} E_{2}^{s, 0}=H^{*}\left(Z_{p} ; Z_{p}\right)$. In particular, $E_{2}^{1,0}=Z_{2}$ and $\phi_{1}$ must be an isomorphism. Let $a \in E_{2}^{1,0}$ be a generator, then $a^{\prime}=\phi_{1}(a)$ is a generator of $H^{1}\left(X / Z_{p}\right)$. By (2.8), $x=\beta(a)$ is a generator of $E_{2}^{2,0}$ and we have $\phi_{2}(x)=\phi_{2} \circ \beta(a)=\beta \circ \phi_{1}(a)=\beta\left(a^{\prime}\right) \neq 0$ by (2.2). Hence $\phi_{2}$ must also be an isomorphism. As $a$ and $x$ generate $E_{2}^{*, 0}$, it follows that

$$
\phi_{s}: E_{2}^{s, 0} \rightarrow H^{s}\left(X / Z_{p}\right)
$$

is an isomorphism for all $1 \leqq s \leqq 2 n+1$. Since $\pi^{*} \circ \phi_{1}(a)=0$, we have $\pi^{*} \circ \phi_{2}(x)=\pi^{*} \circ \beta \circ \phi_{1}(a)=\beta \circ \pi^{*} \circ \phi_{1}(a)=0$. But $\phi_{1}(a)$ and $\phi_{2}(x)$ generate the ring $H^{*}\left(X / Z_{p}\right)$ according to $(2.2)$; it follows that

$$
\pi^{*}: H^{s}\left(X / Z_{p}\right) \rightarrow H^{s}(X)
$$

is trivial for all $s \geqq 1$. Notice that (2) implies that $E_{r}^{s, 0}$ has no cobounding elements for all $r \geqq 2$ and $1 \leqq s \leqq 2 n+1$. In particular we have

$$
d_{s+1}: E_{s+1}^{0, s} \rightarrow E_{s+1}^{s+1,0} \text { is trivial for all } 1 \leqq s \leqq 2 n .
$$

To see the consequence of (3), consider the Smith exact sequences. Following the notations of $[1, \mathrm{p} .41]$, these are

$$
\begin{aligned}
& \cdots \rightarrow H^{s}\left(X / Z_{p}\right) \stackrel{\pi^{*}}{\rightarrow} H^{s}(X) \rightarrow H^{s}(\tau) \rightarrow H^{s+1}\left(X / Z_{p}\right) \rightarrow \cdots, \\
& \cdots \rightarrow H^{s}(\tau) \rightarrow H^{s}(X) \stackrel{\mu}{\longrightarrow} H^{s}\left(X / Z_{p}\right) \rightarrow H^{s+1}(\tau) \rightarrow \cdots .
\end{aligned}
$$

It is known [1] that $\pi^{*} \circ \mu=\sigma^{*}$; hence (3) implies that

$$
\sigma^{*}: H^{s}(X) \rightarrow H^{s}(X) \text { is trivial for all } s \geqq 1 \text {. }
$$

Now we proceed to prove by induction that $H^{s}(X)=0$ for all $1 \leqq s \leqq 2 n$. By exactness of (1), we have $E_{2}^{0,1}=H^{1}(X)^{0}=0$; hence $H^{1}(X)=0$ by $(7)$ and Lemma 2.9. Suppose it has been shown that $H^{i}(X)=0$ for all $1 \leqq i<s \leqq 2 n$. It is easily seen that this implies that the differentials $d_{r}: E_{r}^{0, s} \rightarrow E_{r}^{r, s-r+1}$ are trivial for all $2 \leqq r<s+1$ are $r>s+1$. This together with (4) gives $E_{2}^{0, s}=E_{\infty}^{0, s}$. By (2), we have $E_{\infty}^{s, 0}=\operatorname{Im} \phi_{s}=H^{s}\left(S / Z_{p}\right)$. As $\operatorname{dim} H^{s}\left(X / Z_{p}\right)=\sum_{i=0} E_{\infty}^{s-i, i}$, we have $E_{\infty}^{0, s}=0$. Thus we obtain $E^{0, s}=H^{s}(X)^{0}=0$. Applying Lemma 2.9 again, we obtain $H^{s}(X)=0$.

Next we take up the case $s=2 n+1$. As before, $H^{i}(X)=0$ for all $1 \leqq i \leqq 2 n$ implies that $d_{r}: E_{r}^{0,2 n+1} \rightarrow E_{r}^{r, 2 n+2-r}$ is trivial for all $2 \leqq r<2 n+2$ and $r>2 n+2$. Hence we have $E_{2}^{0,2 n+1}=E_{2 n+2}^{0,2 n+1}$ and $E_{2 n+3}^{0,2 n+1}=E_{\infty}^{0,2 n+1}=0$, where the last equation holds because $H^{2 n+1}\left(X / Z_{p}\right)=E_{\infty}^{2 n+1,0}$ by (2). Similarly, we have $E_{2}^{n+2,0}=E_{2 n+2}^{2 n+2,0}$ and $E_{2 n+3}^{2 n+2,0}=E_{\infty}^{2 n+2,0}=0$. (Since $H^{2 n+2}\left(X / Z_{p}\right)=0$.) But by definition, $E_{2 n+3}^{2 n+2,0}=E_{2 n+2}^{2 n+2,0} / \operatorname{Im}\left(E_{2 n+2}^{0,2 n+1} \rightarrow^{d_{2 n+2}} E_{2 n+2}^{2 n+2,0}\right)$ and $E_{2 n+3}^{0,2+1}$ $=\operatorname{ker}\left(E_{2 n+2}^{0,2 n+1} \rightarrow{ }^{d_{2 n+2}} E_{2 n+2}^{2 n+2,0}\right)$. It follows that $d_{2 n+2}: E_{2 n+2}^{0,2 n+1} \rightarrow E_{2 n+2}^{2 n+2}$, is an 
somorphism. Thus we obtain $E_{2}^{0,2 n+1}=E_{2 n+2}^{0,2 n+1} \cong E_{2 n+2}^{2 n+2,0}=E_{2}^{2 n+2,0}=Z_{p}$. By (7), (2.7) and Lemma 2.10, we obtain $\operatorname{dim} H^{2 n+1}\left(X / Z_{p}\right) \leqq p-1$.

For $s>2 n+1$, let us consider the exact sequences (5) and (6). It is not hard to see [1] that the composition map $H^{s}(X) \rightarrow H^{s}(\tau)$ and $H^{s}(\tau) \rightarrow H^{s}(X)$ is $\tau^{*}$. (5) and (6) then imply that $\tau^{*}: H^{s}(X) \rightarrow H^{s}(X)$ is an epimorphism for all $s>2 n+1$. It follows that $H^{s}(X)=\tau^{*} H^{s}(X)=\cdots=\tau^{* p^{-1}} H^{s}(X)=\sigma^{*} H^{s}(X)=0$ by (7).

Finally let us return to $H^{2 n+1}\left(X / Z_{p}\right)$. As we have now shown that $\operatorname{dim} H^{*}(X)$ $<\infty$, the Euler-characteristic formula of E. E. Floyd [1, p. 40]

$$
\sum_{s=0}^{\infty}(-1)^{s} \operatorname{dim} H(X)=p \sum_{s=0}^{\infty}(-1)^{s} \operatorname{dim} H^{s}\left(X / Z_{p}\right)
$$

can be applied. In our case, this reduces to

$$
1-\operatorname{dim} H^{2 n+1}(X)=0
$$

or $H^{2 n+1}(X)=Z_{p}$. This completes the proof of (2.6).

3. Cohomology covering spaces and lifting of actions. It is a well-known fact that the real projective $n$-space admits the $n$-sphere as its two-folded covering space. The purpose of this section is to give..a construction which among other things will insure that every cohomology real projective $n$-sphere admits a cohomology $n$-sphere $\bmod 2$ as its two-folded covering space. Throughout this section, cohomology always has $Z_{p}$ as coefficient group with no distinction between $p$ being even or odd, $Y$ is a fixed connected space and $a \in H^{1}(Y)$ is a fixed nonzero element.

Let $f: Y \times Y \rightarrow Z_{p}$ be a 1-cocycle representing $a$, then there exists an open covering $\mathscr{V}$ of $Y$ such that

$$
f\left(y_{0}, y_{2}\right)=f\left(y_{0}, y_{1}\right)+f\left(y_{1}, y_{2}\right) \text { whenever } y_{0}, y_{1}, y_{2} \in V \in \mathscr{V} \text {. }
$$

By a $\mathscr{V}$-chain we mean a finite sequence $\left(y_{i}\right)_{i=0}^{n}$ of points of $Y$ such that $\left\{y_{i-1}, y_{i}\right\} \in V_{i} \in \mathscr{V}$ for all $i=1,2, \cdots, n$. Let $b \in Y$ be a fixed base point. By a $\mathscr{V}$-chain with base point $b$ we mean a $\mathscr{V}$-chain $\left(y_{i}\right)_{i=0}^{n}$ such that $y_{0}=b$. The set of all $\mathscr{V}$-chains with base point $b$ is denoted by $\chi \cdot\left(y_{i}\right)_{i=0}^{n},\left(y_{j}^{\prime}\right)_{j=0}^{m} \in \chi$ are said to be equivalent if

and

$$
\begin{gathered}
y_{n}=y_{m}^{\prime} \\
\sum_{i=1}^{n} f\left(y_{i-1}, y_{i}\right)=\sum_{j=1}^{m} f\left(y_{j-1}^{\prime}, y_{j}^{\prime}\right) .
\end{gathered}
$$

The quotient set under this equivalence relation is denoted by $X$ and the equivalence class of $\left(y_{i}\right)_{i=0}^{n} \in \chi$ is denoted by $\left[y_{i}\right]_{i=0}^{n}$. The function $\pi: X \rightarrow Y$ given by $\pi\left(\left[y_{i}\right]_{i=0}^{n}\right)=y_{n}$ is clearly well defined.

Now we topologize $X$ as follows. Let $x=\left[y_{i}\right]_{i=0}^{n} \in X$ and $\mathscr{B}\left(y_{n}\right)$ be a base of neighborhoods of $y_{n}$ such that every $B\left(y_{n}\right) \in \mathscr{B}\left(y_{n}\right)$ is contained in some $V \in \mathscr{V}$. To each $B\left(y_{n}\right) \in \mathscr{B}\left(y_{n}\right)$, define 


$$
\begin{aligned}
B^{*}(x)= & \left\{\left[y_{j}^{\prime}\right]_{j=0}^{m} \in X \mid y_{m}^{\prime} \in B\left(y_{n}\right)\right. \text { and } \\
& \left.\sum_{i=1}^{n} f\left(y_{i-1}, y_{i}\right)+f\left(y_{n}, y_{m}^{\prime}\right)+\sum_{j=1}^{m} f\left(y_{j}^{\prime}, y_{j-1}^{\prime}\right)=0\right\} .
\end{aligned}
$$

It is easily verified that a Hausdorff topology is defined on $X$ with $\mathscr{B}(x)$ $=\left\{B^{*}(x) \mid B\left(y_{n}\right) \in \mathscr{B}\left(y_{n}\right)\right\}$ as a base of neighborhoods at $x$ and that $\pi: X \rightarrow Y$ is a continuous map. In fact, $\pi$ maps every $B^{*}(x)$ homeomorphically onto $B\left(y_{n}\right)$ hence it is even a local homeomorphism. In particular, there exists an open covering $\mathscr{V}^{*}$ of $X$ such that every $V^{*} \in \mathscr{V}^{*}$ is mapped homeomorphically onto some $V \in \mathscr{V}$ by $\pi$.

LEMMA 3.4. For each $y \in Y, \pi^{-1}(y)$ has exactly p points.

Proof. It suffices to consider the case $y=b$. The function $\phi: \pi^{-1}(b) \rightarrow Z_{p}$ given by $\phi\left(\left[y_{i}\right]_{i=0}^{n}\right)=\sum_{i=1}^{n} f\left(y_{i-1}, y_{i}\right)$, where $\left[y_{i}\right]_{i=0}^{n} \in \pi^{-1}(b)$, is clearly injective. Moreover, $\operatorname{Im} \phi \subset Z_{p}$ is a subgroup; hence we have either $\operatorname{Im} \phi=0$ or $\operatorname{Im} \phi=Z_{p}$. If $\operatorname{Im} \phi=0$, we define a 0 -cochain $g: Y \rightarrow Z_{p}$ by $g(y)=\sum_{i=1}^{n} f\left(y_{i-1}, y_{i}\right)$ where $\left(y_{i}\right)_{i=0}^{n}$ is any $\mathscr{V}$-chain with base point $b$ such that $y_{n}=y$. Such a $\mathscr{V}$-chain exists (since $Y$ is connected) and $g$ is well defined. Now if $y, y^{\prime} \in V \in \mathscr{V}$, we have $g\left(y^{\prime}\right)$ $-g(y)=f\left(y, y^{\prime}\right)$ since we can represent $g\left(y^{\prime}\right)$ by $\sum_{i=1}^{n} f\left(y_{i-1}, y_{i}\right)+f\left(y, y^{\prime}\right)$. But this means $f-\delta g$ has empty support, contradicting the assumption that $a \neq 0$.

Notice that (3.4) also implies that $X$ is compact.

By a $\mathscr{V}^{*}$-chain we mean a finite sequence $\left(x_{i}\right)_{i=0}^{n}$ of points of $X$ such that that $\left\{x_{i-1}, x_{i}\right\} \in V_{i}^{*} \in \mathscr{V}^{*}$ for all $i=1,2, \cdots, n$. A $\mathscr{V}^{*}$-chain $\left(x_{i}\right)_{i=0}^{n}$ is said to cover a $\mathscr{V}$-chain $\left(y_{i}\right)_{i=0}^{n}$ (or $\left(y_{i}\right)_{i=0}^{n}$ is covered by $\left.\left(x_{i}\right)_{i=0}^{n}\right)$ if $\pi\left(x_{i}\right)=y_{i}$ for all $i=0,1, \cdots, n$. The function $h: X \rightarrow Z_{p}$ given by

$$
h\left(\left[y_{i}\right]_{i=0}^{n}\right)=-\sum_{i=1}^{n} f\left(y_{i-1}, y_{i}\right)
$$

is clearly well defined having the property that

$$
h\left(x_{0}\right)-h\left(x_{n}\right)=\sum_{i=1}^{n} f\left(y_{i-1}, y_{i}\right)
$$

whenever a $\mathscr{V}^{*}$-chain $\left(x_{i}\right)_{i=0}^{n}$ covers a $\mathscr{V}$-chain $\left(y_{i}\right)_{i=0}^{n}$. The following lemma is immediate.

LEMMA 3.7. (Chain lifting property and monodromy property.) Given a $\mathscr{V}$ chain $\left(y_{i}\right)_{i=0}^{n}$ and a point $x \in \pi^{-1}\left(y_{0}\right)$, there exists a unique $\mathscr{V}^{*}$-chain $\left(x_{i}\right)_{i=0}^{n}$ covering $\left(y_{i}\right)_{i=0}^{n}$ with $x_{0}=x$. Given two $\mathscr{V}$-chains $\left(y_{i}\right)_{i=0}^{n}$ and $\left(y_{j}^{\prime}\right)_{j=0}^{m}$ with $y_{0}=y_{0}^{\prime}$ and $y_{n}=y_{m}^{\prime}$, two $\mathscr{V}^{*}$-chains $\left(x_{i}\right)_{i=0}^{n}$ and $\left(x_{j}^{\prime}\right)_{j=0}^{m}$ covering $\left(y_{i}\right)_{i=0}^{n}$ and $\left(y_{j}^{\prime}\right)_{j=0}^{m}$ respectively with $x_{0}=x_{0}^{\prime}$, then $x_{n}=x_{m}^{\prime}$ if and only if $\sum_{i=1} f\left(y_{i-1}, y_{i}\right)$ $=\sum_{j=1} f\left(y_{j-1}^{\prime}, y_{j}^{\prime}\right)$. 
LEMMA 3.8. There exists a free action of $Z_{p}$ on $X$ such that $X / Z_{p}=Y$ and $\pi$ coincides with the canonical projection.

Proof. Define a map $S: \pi^{-1}(b) \rightarrow \pi^{-1}(b)$ by $S(x)=\phi^{-1}(\phi(x)+1)$, where $x \in \pi^{-1}(b)$ and $\phi: \pi^{-1}(b) \rightarrow Z_{p}$ is the function defined in Lemma 3.4. Extend $S$ to a map $X \rightarrow X$ as follows. Let $x=\left[y_{i}\right]_{i=0}^{n} \in X$ be an arbitrary point of $X$. Choose a $\mathscr{V}^{*}$-chain $\left(x_{i}\right)_{i=0}^{n}$ covering $\left(y_{i}\right)_{i=0}^{n}$ with $x_{0}=S([b])$, where $[b] \in \pi^{-1}(b)$ is the class of the $\mathscr{V}$-chain $(b, b)$; then define $S(x)=x . S$ is well defined in view of Lemma 3.8 and it is easily verified to be a periodic map on $X$ of period $p$ having no fixed point. Later we shall refer to $S$ as the deck-transformation.

Lemma 3.9. The homomorphism $\pi^{*}: H^{1}(Y) \rightarrow H^{1}(X)$ takes a into 0 .

Proof. In fact, $\pi^{*}(a)$ contains the coboundary $\delta h$, where $h$ is the 0 -cochain defined by (3.5).

LEMMA 3.10. $X$ is connected.

Proof. It is not hard to see that if $X$ is not connected, then it has exactly $p$ components $X_{1}, \cdots, X_{p}$ and every $\pi \mid X_{i}: X_{i} \rightarrow Y$ is a homeomorphism. It follows that $\pi^{*}(a) \neq 0$, contrary to (3.9).

Collecting (3.1) through (3.10), we have thus proved

THEOREM 3.11. Let $Y$ be a connected space and $a \in H^{1}(Y)$ a nonzero element; then there exists a space $X$ and a free action of $Z_{p}$ on $X$ such that $Y=X / Z_{p}$ and $\pi^{*}: H^{1}(Y) \rightarrow H^{1}(X)$ maps a into zero.

Because of the last property of $\pi^{*}$, the space $X$ may be called a cohomology covering space of $Y$ with respect to $a \in H^{1}(Y)$. This space can actually be characterized abstractly. We formulate this in the following way. By a cohomology covering space of $Y$ with respect to $a$, we mean a principal bundle $\left(X, Y, Z_{p}, \pi\right)$ such that $\pi^{*}(a)=0$, where $Y$ is a connected space and $a \in H^{1}(Y)$ is a preassigned nonzero element. We state without proof the following uniqueness theorem.

Theorem 3.12. Let $\left(X, Y, Z_{p}, \pi\right)$ and $\left(X^{\prime}, Y, Z_{p}, \pi^{\prime}\right)$ be two cohomology covering spaces of $Y$ with respect to $a \in H^{1}(Y)$ and $g: Y \rightarrow Y$ a homeomorphism such that $g^{*}(a)=a$, where $g^{*}: H^{1}(Y) \rightarrow H^{1}(Y)$ is the homomorphism induced by $g$. Then there exists a homeomorphism $\tilde{g}: X \rightarrow X^{\prime}$ such that $\pi^{\prime} \circ \tilde{g}=g \circ \pi$. Moreover, let $x \in X$ and $x^{\prime} \in X^{\prime}$ be any two preassigned points such that $\pi^{\prime}\left(x^{\prime}\right)=g \circ \pi(x)$; then $\tilde{g}$ can be chosen in such a way that $\tilde{g}(x)=x^{\prime}$, and it is completely determined by this condition.

Suppose that $G$ is a transformation group on $Y$, a bundle lifting of $G$ is an action of $G$ on $X$ such that each $g \in G$ acts on $X$ as a bundle map $\tilde{g}$ [7] (i.e., it commutes with the deck-transformation), and $\pi \circ \tilde{g}(x)=g \circ \pi(x)$ for all $x \in X$. It follows that a bundle lifting of $G$ defines an action of $Z_{p} \times G$ on $X$. 
THEOREM 3.13. Let $\left(X, Y, Z_{p}, \pi\right)$ be a cohomology covering space of $Y$ with respect to $a \in H^{1}(Y)$ and $G$ a finite transformation group on $Y$. Suppose that $a$ is invariant under $G$ (i.e., $g^{*}(a)=a$ for all $g \in G$ ) and that the fixed point set $F(G ; Y) \neq \varnothing$. Then $G$ has a bundle lifting.

Proof. In view of (3.12), it suffices to consider a particular $X$. This can be obtained by a slight modification of the construction given in the beginning of this section. There exists an open covering $\mathscr{V}$ of $Y$, a 1-cocycle $f: Y \times Y \rightarrow Z_{p}$ representing $a$ and to each $g \in G$ a 0 -cochain $k_{g}: Y \rightarrow Z_{p}$ such that (i) $g V \in \mathscr{V}$ for all $g \in G$ and $V \in \mathscr{V}$, (ii) (3.1) holds true and (iii) $f\left(y, y^{\prime}\right)-f\left(g(y), g\left(y^{\prime}\right)\right)$ $=k_{g}(y)-k g\left(y^{\prime}\right)$ whenever $y, y^{\prime} \in V \in \mathscr{V}$. Let $\chi$ be the set of all $\mathscr{V}$-chains with base point $b$, where $b$ is chosen as a point in $F(G ; Y)$. By (i), every $g \in G$ induces a function $g: \chi \rightarrow \chi$ defined as $g\left(\left(y_{i}\right)_{i=0}^{n}\right)=\left(g\left(y_{i}\right)\right)_{i=0}^{n}$. Define an equivalence relation in $\chi$ as (3.2). Condition (iii) insures that each $g$ takes equivalent $\mathscr{V}$-chains into equivalent $\mathscr{V}$-chains and hence induces a map $\tilde{g}: X \rightarrow X$. The rest of the theorem is obvious.

4. Fixed point sets of actions of $Z_{p}$ on cohomology real projective spaces or cohomology lens spaces. With the machineries built up in the previous two sections, it is now easy to establish two of the main theorems of this paper.

THEOREM 4.1. If $Z_{2}$ acts on a cohomology real projective $n$-space, then the fixed point set $F$ is either empty or it has at most two components. If $F$ has $k$ components $C_{1}, \cdots, C_{k}, 1 \leqq k \leqq 2$, then each $C_{i}$ is a cohomology real projective $n_{i}$-space, $i=1, \cdots, k$ and

$$
\sum_{i=1}^{k} n_{i}=n-k+1
$$

Proof. Let $Z_{2}$ act on a cohomology real projective $n$-space $Y$, that is, an involution $T: Y \rightarrow Y$ and suppose that $F\left(Z_{2} ; Y\right)=F \neq \varnothing$. According to (3.11), there is a cohomology covering space $X$ with respect to $a$, where $a \in H^{1}\left(Y ; Z_{2}\right)$ is a generator. As $X$ is connected, we know that $X$ is a cohomology $n$-sphere mod 2 by (2.5). Since $F \neq \varnothing$ and $a$ is necessarily invariant under $T^{*}, T$ can be lifted to an involution $\tilde{T}: X \rightarrow X$ which commutes with the deck-transformation $S$ on $X$ (see Lemma 3.8). $\tilde{T}$ and $S$ together then define an action of $Z_{2} \times Z_{2}$ on $X$. Consider the subgroups in $Z_{2} \times Z_{2}$ generated by $(S, 1)(1, \tilde{T})$ and $(S, \tilde{T})$, their fixed point sets $F_{0}, F_{1}$ and $F_{2}$ respectively. By the well-known theorem of P. A. Smith [1], $F_{i}$ is a cohomology $n_{i}$-sphere $\bmod 2, i=0,1,2$. Moreover, by a theorem of P. A. Smith [6] and A. Borel [1, p. 175], we have the relation that $\sum_{i=0}^{2}\left(n_{i}+1\right)$ $=n+1$. Obviously, we have $n_{0}=-1$ since $S$ acts freely on $X$. Hence the equation reduces to $n_{1}+n_{2}=n-1$. It is not hard to see that $\pi\left(F_{1}\right)$ and $\pi\left(F_{2}\right)$, if not empty, are precisely the components of $F\left(Z_{2} ; Y\right)$. Now $S$ acts on $F_{1}$ and $F_{2}$, 
freely of course; therefore by $(2.3) \pi\left(F_{1}\right)$ and $\pi\left(F_{2}\right)$ are cohomology real projective $n_{i}$-spaces, $i=1,2$. This completes the proof of (4.1).

In precisely the same way, one can prove

THEOREM 4.2. If $Z_{p}(p \neq 2)$ acts on a cohomology lens $(2 n+1)$-space $\bmod p$, then the fixed point set $F$ is either empty or it has at most $p$ components. If $F$ has $k$ components $C_{1}, \cdots, C_{k}, 1 \leqq k \leqq p$, then each $C_{i}$ is a cohomology lens $\left(2 n_{i}+1\right)$-space $\bmod p, i=1,2, \cdots, k$ and

$$
\sum_{i=1}^{k} n_{i}=n-k+1 .
$$

5. Cohomology complex projective spaces. We now turn to actions of the circle group $S^{1}$. An action of $S^{1}$ on a space $X$ is said to have finite orbit type [1] if the set $\left\{S_{x}^{1} \subset S^{1} \mid x \in X\right\}$ is finite, i.e., if there is only a finite number of distinct isotropic subgroups. We shall need the notion of universal bundle and classifying space $\left[1\right.$, p. 52]. The universal bundle for $S^{1}$ is the space $\bigcup_{n=1}^{\infty} S^{2 n+1}$ and the classifying space for $S^{1}$ is the infinite dimensional complex projective space $C P^{\infty}$. These spaces are not compact (in fact, not even locally compact) and hence do not fit into the cohomology we are using now. As usual, this complication can be avoided by confining ourselves to spaces $X$ on which $S^{1}$ acts to have finite cohomology dimension over $Z\left[1\right.$, p. 6] (notation: $\operatorname{dim}_{z} X<\infty$ ). Then by taking a sufficiently large $N \gg \operatorname{dim}_{Z} X$, we may "regard" $S^{2 N+1}$ and $C P^{N}$ (complex projective $N$-space) as the universal bundle $E_{S^{1}}$ and the classifying space $B_{S^{1}}$ for the group $S^{1}$ (see [1, p. 52] for detailed explanation). This convention shall be used from now on. Throughout the rest of this paper, cohomology will always have the group of integers $Z$ as coefficient domain unless otherwise stated. By a cohomology complex projective $n$-space we mean a space $Y$ whose integral cohomology ring $H^{*}(Y)$ is given by

$$
H^{*}(Y)=Z[x] /\left(x^{n+1}\right), \text { degree } x=2,
$$

where $Z[x]$ is the polynomial ring over $Z$ and $\left(x^{n+1}\right)$ is the ideal generated by $x^{n+1}$.

Proposition 5.2. If $S^{1}$ acts freely on an integral cohomology $(2 n+1)-$ sphere $X$ and if $\operatorname{dim}_{z} X<\infty$, then $X / S^{1}$ is a cohomology complex projective $n$-space.

Proof. There exists a spectral sequence $\left(E_{r}\right)[1]$ whose $E_{2}$-term is given by $E_{2}^{s, t}=H^{s}\left(B_{S^{1}} ; H^{t}(X)\right)$ and whose $E_{\infty}$-term is associated with $H^{*}\left(X / S^{1}\right)$ (up to certain dimension). The assertion follows readily from the Gysin sequence of $\left(E_{r}\right)$ and the fact that $H^{*}\left(B_{S^{1}}\right)$ is given by

$$
H^{*}\left(B_{S^{1}}\right)=Z[x] /\left(x^{N+1}\right) \text {, degree } x=2,2 N+1>\operatorname{dim}_{Z} X .
$$


THEOREM 5.4. If $S^{1}$ acts freely on $X$ such that $X / S$ is a cohomology complex projective $n$-space and $\operatorname{dim}_{z} X / S^{1}<\infty$. If moreover $\pi^{*}: H^{2}\left(X / S^{1}\right) \rightarrow H^{2}(X)$ is trivial, then $X$ is an integral cohomology $(2 n+1)$-sphere and $\operatorname{dim}_{\mathrm{Z}} X<\infty$.

Proof. We shall only prove the case $n>0$. Since $S^{1}$ acts freely on $X$, $\pi: X \rightarrow X / S^{1}$ is a fiber map by a theorem of A. Gleason [4]. This implies easily that $\operatorname{dim}_{\mathrm{z}} X<\infty$. We can therefore consider the spectral sequence $\left(E_{r}\right)$ of (5.2) where we have $E_{2}^{s, t}=H^{s}\left(B_{S^{1}} ; H^{t}(X)\right)=H^{s}\left(B_{S^{1}}\right) \otimes H^{t}(X)\left(H^{*}\left(B_{S^{1}}\right)\right.$ has no torsion). In particular, we have

$$
E_{2}^{s, t}=0 \text { whenever } s \text { is odd. }
$$

Consider the sequence

$$
0 \rightarrow E_{2}^{1,0} \stackrel{\phi_{1}}{\longrightarrow} H^{1}\left(X / S^{1}\right) \stackrel{\pi^{*}}{\longrightarrow} E_{2}^{0,1} \stackrel{d_{2}}{\longrightarrow} E_{2}^{2,0} \stackrel{\phi_{2}}{\longrightarrow} H^{2}\left(X / S^{1}\right) \stackrel{\pi^{*}}{\longrightarrow} E_{2}^{0,2} .
$$

In general, the last place of this sequence is not exact but only satisfies $\operatorname{Im} \phi_{2}$ $\subset \operatorname{ker} \pi^{*}$ and $\operatorname{ker} \pi^{*} / \operatorname{Im} \phi_{2}=E_{\infty}^{1,1}$. But in our case $E_{\infty}^{1,1}=0$ by (1). Taking account of the fact that $H^{1}\left(X / S^{1}\right)=0$, we have thus the following exact sequence:

$$
0 \rightarrow E_{2}^{0,1} \stackrel{d_{2}}{\longrightarrow} E_{2}^{2,0} \stackrel{\phi_{2}}{\longrightarrow} H^{2}\left(X / S^{1}\right) \stackrel{\pi^{*}}{\longrightarrow} E_{2}^{0,2} .
$$

Now $\pi^{*}: H^{2}\left(X / S^{1}\right) \rightarrow E_{2}^{0,2}=H^{2}(X)$ is trivial by hypothesis; hence (2) reduces to an exact sequence $0 \rightarrow E_{2}^{0,1} \rightarrow Z \rightarrow{ }^{\phi_{2}} Z \rightarrow 0$. This implies $\phi_{2}: E_{2}^{2,0} \rightarrow H^{2}\left(X / S^{1}\right)$ is an isomorphism and $E_{2}^{0,1}=H^{1}(X)=0$. As $E_{2}^{*, 0}=\sum_{s=0}^{\infty} E_{2}^{s, 0}=H^{*}\left(B_{S^{1}}\right)$, by $(5.3)$ and (5.1) one deduces that

$$
\phi_{s}: E_{2}^{s, 0} \rightarrow H^{s}(X / S)
$$

is an isomorphism for all $1 \leqq s \leqq 2 n+1$. Just as in Theorem 2.6, this fact and $H^{1}(X)=0$ enables one to prove inductively that $H^{s}(X)=0$ for all $1 \leqq s \leqq 2 n$. Again as in (2.6), one then uses this and proceeds to show that $E_{2}^{0,2 n+1}=E_{2 n+2}^{0,2 n+1}$, that $E^{2 n+2,0}=E_{2 n+2}^{2 n+2,0}$ and that $d_{2 n+2}: E_{2 n+2}^{0,2 n+1} \rightarrow E_{2 n+2}^{2 n+2,0}$ is an isomorphism. Hence $H^{2 n+1}(X)=E_{2}^{0,2 n+1} \cong E_{2}^{2 n+2,0}=Z$.

For higher dimensional groups, of course, no special cohomology theory is available here. Instead, we propose to prove by induction that $H^{2 n+k}(X)=0$ for all $k \geqq 2$. We have seen that $\theta_{0}=d_{2 n+2}: E_{2}^{0,2 n+1} \rightarrow E_{2}^{2 n+2,0}$ is an isomorphism. This can be described as follows. Let $a$ be a generator of $H^{2}\left(B_{S^{1}}\right)$ and 1 denote the generator of $H^{0}\left(B_{S^{1}}\right)$ as well as that of $H^{0}(X)$. Consider $a^{n+1} \otimes 1 \in E_{2}^{2 n+2,0}$, regard it as in $E_{2 n+2}^{2 n+2,0}$; then there exists uniquely an element $b \in H^{2 n+1}(X)$ such that $1 \otimes b \in E_{2}^{0,2 n+1}$, considered as in $E_{2 n+2}^{0,2 n+1}$, satisfies $d_{2 n+2}(1 \otimes b)=a^{n+1} \otimes 1$, and $\theta_{0}$ is entirely determined by the relation $\theta_{0}(1 \otimes b)=a^{n+1} \otimes 1$.

Let $Z\left(E_{r}^{s, t}\right)$ be the cocycles of $E_{r}^{s, t}, \mu_{r}^{s, t}: Z\left(E_{r}^{s, t}\right) \rightarrow E_{r+1}^{s, t}$ the projection and $j_{r}^{s, t}: Z\left(E_{r}^{s, t}\right)$ $\rightarrow E_{r}^{s, t}$ the inclusion. We agree that if we write $\mu_{r}^{s, t}: E_{r}^{s, t} \rightarrow E_{r+1}^{s, t}$, it is tacitly assumed that $Z\left(E_{r}^{s, t}\right)=E_{r}^{s, t}$. Similarly, if we write $j_{r}^{s, t}: E_{r+1}^{s, t} \rightarrow E_{r}^{s, t}$, it is tacitly assumed 
that $Z\left(E_{r}^{s-r, t+r-1}\right)=E_{r}^{s-r, t+r-1}$ and $E_{r+1}^{s, t}$ has been identified with $Z\left(E_{r}^{s, t}\right)$. It is easily verified that we have the following diagram:

$$
\begin{aligned}
& E_{2}^{0,2 n+2} \stackrel{j_{2}^{0,2 n+2}}{\longleftarrow} E_{3}^{0,2 n+2}=E_{2 n+3}^{0,2 n+2}=E_{2 n+4}^{0,2 n+2}=E_{\infty}^{0,2 n+2}=0
\end{aligned}
$$

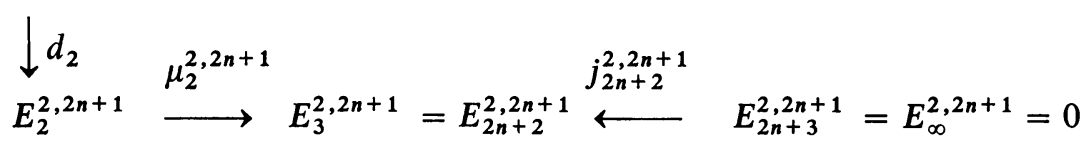

$$
\begin{aligned}
& \downarrow d_{2 n+2} \\
& E_{2}^{2 n+4,0} \quad=\quad E_{2 n+2}^{2 n+4,0} \text {. }
\end{aligned}
$$

In this diagram, $\operatorname{ker} d_{2}=\operatorname{Im} j_{2}^{0,2 n+2}=0$. Define $\theta_{2}: E_{2}^{2,2 n+1} \rightarrow E_{2}^{2 n+4,0}$ by $\theta_{2}=d_{2 n+2} \mu_{2}^{2,2 n+1}$. Since $\operatorname{ker} d_{2 n+2}=\operatorname{Im} j_{2 n+2}^{2,2 n+1}=0$, we have $\operatorname{ker} \theta_{2}=\operatorname{ker} \mu_{2}^{2,2 n+1}$ $=\operatorname{Im} d_{2}$. In other words, $0 \rightarrow E_{2}^{0,2 n+2} \rightarrow^{d_{2}} E_{2}^{2,2 n+1} \rightarrow^{\theta_{2}} E_{2}^{2 n+4,0}$ is exact. Now consider $a \otimes b \in E_{2}^{2,2 n+1}$, we have $\theta_{2}(a \otimes b)=d_{2 n+2}((a \otimes 1)(1 \otimes b))=(a \otimes 1)$ $d_{2 n+2}(1 \otimes b)$ because $d_{2 n+2}(a \otimes 1)=0$. That is, we have the following commutative diagram:

$$
\begin{array}{rll}
0 \rightarrow E_{2}^{0,2 n+2} \stackrel{d_{2}}{\longrightarrow} E_{2}^{2,2 n+1} & \stackrel{\theta_{2}}{\longrightarrow} E_{2}^{2 n+4} 0 \\
\gamma_{a} \otimes 1 & \uparrow & \uparrow \gamma_{a} \otimes 1 \\
& E_{2}^{0,2 n+1} \stackrel{\theta_{0}}{\longrightarrow} E_{2}^{2 n+2,0},
\end{array}
$$

where $\gamma_{a}: H^{*}\left(B_{S^{1}}\right) \rightarrow H^{*}\left(B_{S^{1}}\right)$ is the multiplication by $a \in H^{2}\left(B_{S^{1}}\right)$. Since $\gamma_{a}$ and $\theta_{0}$ are isomorphisms, $\theta_{2}$ must be an isomorphism and hence the exactness of the upper row implies that $H^{2 n+2}(X)=E_{2}^{0,2 n+2}=0$.

Suppose it has been proved that $H^{2 n+i}(X)=0$ for all $2 \leqq i<k$. In a similar manner as above, one can show that there exists a commutative diagram

for $k$ even and

$$
\begin{aligned}
0 \longrightarrow E_{2}^{0,2 n+k} \longrightarrow E_{2}^{k, 2 n+1} & \stackrel{\theta_{k}}{\longrightarrow} E_{2}^{2 n+k+2,0} \\
\gamma_{a}^{k / 2} \otimes 1 \uparrow & \\
E_{2}^{0,2 n+1} & \stackrel{\theta_{0}}{\longrightarrow} E_{2}^{2 n+2,0} \otimes 1
\end{aligned}
$$

$$
\begin{aligned}
E_{2}^{k-1,2 n+1} \stackrel{\theta_{k}}{\longrightarrow} & E_{2}^{2 n+k+1,0} \longrightarrow E_{2}^{0,2 n+k} \longrightarrow 0 \\
\gamma_{a}^{(k-1) / 2} \otimes 1 \uparrow_{\gamma_{a}^{(k-1) / 2} \otimes 1}^{\longrightarrow} & \\
E_{2}^{0,2 n+1} \stackrel{\theta_{0}}{\longrightarrow} & E_{2}^{2 n+2,0}
\end{aligned}
$$

for $k$ odd with exact upper rows. In either case, one concludes that $H^{2 n+k}(X)$ $=E_{2}^{0,2 n+k}=0$. The proof of (5.4) is completed. 
6. Lifting of an action in a principal bundle $\left(X, Y, S^{1}, \pi\right)$.

Proposition 6.1. Let $Y$ be a space and a an element of $H^{2}(Y)$, then there exists a principal bundle $\left(X, Y, S^{1}, \pi\right)$ such that $\pi^{*}: H^{2}(Y) \rightarrow H^{2}(X)$ maps a into zero.

Proof. Represent $Y$ as the inverse limit of an inverse system $\left\{Y_{m}, \phi_{m}^{m^{\prime}}\right\}$ of triangulable spaces [3]. Let $\phi_{m}: Y \rightarrow Y_{m}$ be the projection. By the continuity property of cohomology, there exists an index $m$ and $a_{m} \in H^{2}\left(Y_{m}\right)$ such that $\phi_{m}^{*}\left(a_{m}\right)=a$. Consider the principal bundle $\left(S^{2 N+1}, C P^{N}, S^{1}, p\right)$, where $N$ is so chosen that $2 N+1>\operatorname{dim} Y_{m}$. According to the standard obstruction theory [5], there exists a map $g: Y_{m} \rightarrow C P^{N}$ such that $g^{*}(x)=a_{m}$, where $x \in H^{2}\left(C P^{N}\right)$ is a generator. We have therefore a map $f: Y \rightarrow C P^{N}\left(f=g \circ \phi_{m}\right)$ such that $f^{*}(x)=a$. The bundle induced by $f$ has all the desired properties stated in (6.1).

Now suppose that an action of $S^{1}$ is given on the base space $Y$ in a principal bundle $\left(X, Y, S^{1}, \pi\right)$. The notion of bundle lifting is defined in the same way as in $\S 3$. More precisely, let $\beta: S^{1} \times X \rightarrow X$ represent the action of the structural group $S^{1}$ on $X$ and $\alpha: S^{1} \times Y \rightarrow Y$ the given action of $S^{1}$ on $Y$. Then a bundle lifting $\tilde{\alpha}$ of $\alpha$ is a map $\tilde{\alpha}: S^{1} \times X \rightarrow X$ which defines an action of $S^{1}$ on $X$ and satisfying the conditions (i) $\pi \circ \tilde{\alpha}(g, x)=\alpha(g, \pi(x))$ and (ii) $\tilde{\alpha}\left(g_{1}, \beta\left(g_{2}, x\right)\right)=$ $\beta\left(g_{2}, \tilde{\alpha}\left(g_{1}, x\right)\right)$. The following result is essentially due to T. E. Stewart [8].

Proposition 6.2. Let $\left(X, Y, S^{1}, \pi\right)$ be a principal bundle and $\alpha: S^{1} \times Y \rightarrow Y$ an action of $S^{1}$ on $Y$. If $H^{1}(Y)=0$, then $\alpha$ has a bundle lifting.

Proof. Let $R$ be the additive group of reals and $\varphi: R \rightarrow S^{1}$ the usual exponential map. The map $\alpha^{\prime}: R \times Y \rightarrow Y$ given by $\alpha^{\prime}(t, y)=\alpha(\varphi(t), y)$ defines an action of $R$ on $Y$. Using the same argument employed in [8, Lemma 3.3], one deduces that $\alpha^{\prime}$ has a bundle lifting $\alpha^{\prime \prime}: R \times X \rightarrow X$. Now to "push" $\alpha^{\prime \prime}$ down to $S^{\mathbf{1}}$, all we have to do is to adjust it in such a way that it becomes periodic. Define a map $g: Y \rightarrow S^{1}$ by the condition that $\alpha^{\prime \prime}(0, x)=\beta\left(g(y), \alpha^{\prime \prime}(1, x)\right)$ for all $x \in \pi^{-1}(y)$. Since $H^{1}(Y)=0$, i.e., $\pi^{1}(Y)=0$, where $\pi^{1}(Y)$ is the Bruschlinsky group [5] of $Y, g$ is homotopic to zero. Hence $g$ can be factored as $g=\varphi \circ h$ by a map $h: Y \rightarrow R$. Define $\hat{\alpha}: R \times X \rightarrow X$ as

$$
\left.\hat{\alpha}(t, x)=\beta(\varphi \operatorname{th}(\pi(x))), \alpha^{\prime \prime}(t, x)\right) \text {. }
$$

It is easily verified that $\hat{\alpha}$ defines an action of $R$ on $X$ satisfying $\hat{\alpha}(0, x)=\hat{\alpha}(1, x)$. Hence the map $\tilde{\alpha}: S^{1} \times X \rightarrow X$ given by

$$
\tilde{\alpha}(\varphi(t), x)=\hat{\alpha}(t, x)
$$

is well defined, it gives an action of $S^{1}$ on $X$ which is a bundle lifting of $\alpha$.

7. Actions of $S^{1}$ on cohomology complex projective spaces. We first prove a proposition that will be needed in the proof of the main theorem and it is also interesting by itself. 
Proposition 7.1. Let $S^{1}$ act on a cohomology complex projective $n$-space $Y$ and assume that $\operatorname{dim}_{Z} Y<\infty$. Then the fixed point set $F$ is nonempty and has at most $n+1$ components.

Proof. The proof is based on the following well-known device. Let $Y_{S^{1}}$ be the orbit space of the diagonal action of $S^{1}$ on $Y \times E_{S^{1}}$, where $E_{S^{1}}$ is taken as the sphere $S^{2 N+1}$ with $2 N+1>\operatorname{dim}_{Z} Y$. There are $[1$, p. 50] natural maps $\pi_{1}: Y_{S^{1}} \rightarrow Y / S^{1}$ and $\pi_{2}: Y_{S^{1}} \rightarrow B_{S^{1}}$ and $\pi_{2}$ is always a fibering with $Y$ as fiber. Consider the spectral sequence $\left(E_{r}\right)$ of $\pi_{2}$ with the rational field $Q$ as coefficient domain. We have $E_{2}^{s, t}=H^{s}\left(B_{S^{1}} ; H^{t}(Y ; Q)\right)$ and the $E_{\infty}$-term is associated with $H^{*}\left(Y_{S^{1}} ; Q\right)$. Since $E_{2}^{s, t}=0$ when either $s$ or $t$ is odd, it is easily seen that $\left(E_{r}\right)$ is trivial. This enables one to compute readily that

$$
\operatorname{dim} H^{k}\left(Y_{S}^{1} ; Q\right)= \begin{cases}0, & \text { if } k \text { is odd } \\ m+1 & \text { if } k=2 m, 0 \leqq m \leqq n \\ n+1 & \text { if } k=2 m, n \leqq m \leqq N\end{cases}
$$

The map $\pi_{1}$ is in general not a fibering but for each $z=\pi(y) \in Y / S^{1}$ we have $\pi^{-1}(z)=S^{2 N+1} / S^{1} y$, where $S^{1} y$ is the isotropic subgroup at $y$. Now suppose that $F=\phi$. Then each $S^{1} y$ is a finite group and the rational cohomology of $S^{2 N+1} / S^{1} y$ is trivial, i.e., $H^{k}\left(S^{2 N+1} / S^{1} y ; Q\right)=0$ for all $1 \leqq k \leqq 2 N$. It follows from the Vietoris mapping theorem that $\pi_{1}^{*}: H^{k}\left(Y_{S^{1}} ; Q\right) \rightarrow H^{k}\left(Y_{S^{1}} ; Q\right)$ is an isomorphism for all $0 \leqq k \leqq 2 N$. In particular, take $k$ even with $\operatorname{dim}_{Z} Y<k \leqq 2 N$, we obtain from (1) that $H^{k}\left(Y / S^{1} ; Q\right) \neq 0$. But this is a contradiction because $\operatorname{dim}_{Q} Y / S^{1} \leqq \operatorname{dim}_{Z} Y / S^{1} \leqq \operatorname{dim}_{Z} Y[1$, p. 111].

Just as $Y_{S^{1}}$, we can form the space $F_{S^{1}}$ which is simply $B_{S^{1}} \times F$. The inclusion $i: F \rightarrow Y$ induces a homomorphism $i^{*}: H^{k}\left(Y_{S^{1}} ; Q\right) \rightarrow H^{k}\left(F_{S^{1}} ; Q\right)$. It is known [1, p. 54] that $i^{*}$ is an isomorphism for all $\operatorname{dim}_{Q} Y<k \leqq 2 N$. Take $k=2 N$, by (1) and the Kunneth formula we obtain $\operatorname{dim} H^{0}(F ; Q) \leqq n+1$.

We now present the last main theorem of this paper.

THEOREM 7.2. Let $S^{1}$ act on a cohomology complex projective $n$-space $Y$. Suppose that $\operatorname{dim}_{\mathrm{Z}} Y<\infty$ and that the action has finite orbit type. Then the fixed point set $F$ is nonempty, it has at most $n+1$ components, say $C_{1}, \cdots, C_{k}$ $1 \leqq k \leqq n+1$, where each $C_{i}$ is a cohomology complex projective $n_{i}$-space, $i=1,2, \cdots, k$, and

$$
\sum_{i=1}^{k} n_{i}=n-k+1
$$

Proof. Let $a \in H^{2}(Y)$ be a generator. By (6.1), there exists a principal bundle $\left(X, Y, S^{1}, \pi\right)$ such that $\pi^{*}: H^{2}(Y) \rightarrow H^{2}(X)$ is trivial and therefore $X$ is an integral cohomology $(2 n+1)$-sphere according to (5.4). Let $\alpha: S^{\mathbf{1}} \times Y \rightarrow Y$ denote the given action and $\beta: S^{1} \times X \rightarrow X$ the action of the structural group. By (6.2), $\alpha$ 
has a bundle lifting $\tilde{\alpha}: S^{1} \times X \rightarrow X$. $\tilde{\alpha}$ and $\beta$ together then define an action $\gamma:\left(S^{1} \times S^{1}\right) \times X \rightarrow X$ of $S^{1} \times S^{1}$ on $X$ by $\gamma\left(\left(g_{1}, g_{2}\right), \tilde{x}\right)=\alpha\left(g_{1}, \beta\left(g_{2}, x\right)\right)$. We claim that $\gamma$ has finite orbit type. Suppose that $\left(g_{1}, g_{2}\right) \in G_{x} \subset S^{1} \times S^{1}$. Let $y=\pi(x)$, then $g_{1} \in G y$. If $G y \neq S^{1}$, i.e., $G y$ is finite, it is easily seen that $G_{x}=G y \times N$, where $N \subset G y$ is a subgroup. Hence there is only a finite number of $G_{x}$ of this type since $\alpha$ has finite orbit type. If $G y=S^{1}$, i.e., $y \in F$, it is easily seen that $G_{x}$ is then of the form $G_{x}=\left\{\left(g, g^{k y}\right) \mid g \in S^{1}\right\}$, where $k y$ is some integer depending on $y$. Moreover, the function $y \rightarrow k y$ is continuous on $F$, hence it must be constant on each component of $F$. By (7.1), $F$ has at most $n+1$ components, say $C_{1}, \cdots, C_{k}$, $1 \leqq k \leqq n+1$. To each $C_{i}, \quad i=1,2, \cdots, k$. Let $k_{i}=k y, \quad y \in C_{i}$ and $H_{i}=$ $\left\{\left(g, g^{k} i\right) \mid g \in S^{1}\right\}$. Then $G_{x}$ must be one of the $H_{i}, i=1,2, \cdots, k$. This proves the assertion that $\gamma$ has finite orbit structure.

Now let $F_{i}$ be the fixed point set of $H_{i}$. By a theorem of E. E. Floyd [1, p. 63], $F_{i}$ is an integral cohomology $m_{i}$-sphere and by dimension parity, $m_{i}$ must be odd, say $m_{i}=2 n_{i}+1, i=1,2, \cdots, k$. It is easily seen that $\beta: S^{1} \times F_{i} \rightarrow F_{i}$ is a free action of $S^{1}$ on $F_{i}$ for which $F_{i} / S^{1}=C_{i}$. By (5.2), $C_{i}$ is a cohomology complex projective $n_{i}$-space, $i=1,2, \cdots, k$. Finally, by a theorem of A. Borel [1, p. 175], we have the relation

$$
\sum_{i=1}^{k}\left(m_{i}-(-1)\right)=(2 n+1)-(-1)
$$

that is,

$$
\sum_{i=1}^{k} n_{i}=n-k+1
$$

\section{BIBLIOGRAPHY}

1. A. Borel et al., Seminar on transformation groups, Annals of Mathematics Studies No. 46, Princeton Univ. Press, Princeton, N. J., 1960.

2. H. Cartan and S. Eilenberg, Homological algebra, Princeton Univ. Press, Princeton, N. J., 1956.

3. S. Eilenberg and N. E. Steenrood, Foundations of algebraic topology, Princeton Univ. Press, Princeton, N. J., 1952.

4. A. Gleason, Spaces with a compact Lie group of transformations, Proc. Amer. Math. Soc. 1 (1950), 35-43.

5. S. T. Hu, Homotopy theory, Academic Press, New York, 1959.

6. P. A. Smith, New results and old problems in finite transformation groups, Bull. Amer. Math. Soc. 66 (1960), 401-415.

7. N. E. Steenrod, The topology of fibre bundles, Princeton Univ. Press, Princeton, N. J., 1951.

8. T. E. Stewart, Lifting the action of a group in a fibre bundle, Bull. Amer. Math. Soc. 66 (1960), 129-132.

University of Pennsylvania,

Philadel Phia, Pennsylvania

UNIVERSITY OF VIRGINIA,

Charlottesville, Virginia 